\title{
GENERALIZED BROADBAND BEAMFORMING USING A MODAL DECOMPOSITION
}

\author{
Michael I. Y. Williams, Thushara D. Abhayapala, and Rodney A. Kennedy \\ Wireless Signal Processing Program, National ICT Australia, \\ and Department of Information Engineering, The Australian National University \\ \{michael.williams, thushara.abhayapala, rodney.kennedy\}aanu.edu.au
}

\begin{abstract}
We propose a new broadband beamformer design technique which produces an optimal beampattern for any set of samples in space and time. The modal subspace decomposition (MSD) technique is based on projecting a desired pattern into the subspace of patterns achievable by a particular set of space-time sampling positions. This projection is the optimal achievable pattern, in the sense that it minimizes the mean-squared error (MSE) between the desired and actual patterns. The main advantage of the technique is versatility as it can produce optimal beamformers for both sparse and dense arrays, non-uniform and asynchronous time sampling, and dynamic arrays where sensors can move throughout space. It can also be applied to any beampattern type, including frequency-invariant and spot pattern design.
\end{abstract}

\section{INTRODUCTION}

\subsection{Motivation and Background}

Beamforming is an important area of research with applications in acoustics, wireless communications, sonar, and radar [1]. The response of a broadband beamformer to farfield sources can be expressed using a beampattern - a 2D function of angle and frequency/wavenumber. A perfect beampattern is designed to reject noise and interfering sources, but depending on sensor positions and time sampling, this desired pattern may not be achievable by a particular array geometry. The beamformer design problem is to find an achievable pattern as close as possible to the desired pattern.

One popular technique for beampattern design is the frequency decomposition method [2]. In this method, the time sequences measured at each sensor are projected into narrowband frequency bins using the Discrete Fourier Transform (DFT). A broadband beampattern can then be created by using established narrowband beamforming techniques within each frequency bin.

One limitation of this technique is that sensors must be fixed, and closely spaced. To avoid spatial aliasing in the highest frequency bin, sensors must be placed no further than half of the corresponding wavelength apart [3,4].

Another limitation is that perfect frequency decomposition would require an infinite length time sequence sampled at the Nyquist rate (twice the highest design frequency). In many applications, however, getting a sequence of reasonable length may be impossible. Consider an environment where source field is changing rapidly - past samples will quickly become useless, and short time sequences must be used to maintain relevance.

National ICT Australia is funded through the Australian Government's Backing Australia's Ability initiative, in part through the Australian Research Council.
On top of these limitations, traditional broadband beamforming techniques suffer from a lack of versatility. Most implementations focus on the design of frequency-invariant beampatterns $[3,4]$, and there is little scope for more complex patterns. They are also generally based on the simple model of a static sensor array in space, sampled synchronously and uniformly throughout time. This can cause problems in real-world applications, such as sensor networks, which may require asynchronous sampling (where different sensors are sampled at different times), or dynamic arrays (where sensors are able to move throughout space).

In this paper, we develop a beamformer design technique, based on a modal subspace decomposition (MSD), which addresses these weaknesses and limitations. The technique is applicable to any desired beampattern (including both frequency-invariant and more complex types), any sampling scheme (including both sparse and dense sampling, non-uniform and asynchronous time sampling, and moving sensors), and always produces the optimal achievable pattern (where optimality is defined as minimizing the mean-square error, or MSE, between the desired and actual patterns).

\section{INTRODUCTION TO BEAMFORMING}

Consider wavefields, $f(\boldsymbol{x}, t)$, in $2 \mathrm{D}$ space and time, generated by integrating some farfield distribution, $F(k, \phi)$, over azimuth angle, $\phi=[-\pi, \pi]$, and some bandlimited range of wavenumber, $k=$ $\left[k_{1}, k_{2}\right][5]$,

$$
f(\boldsymbol{x}, t)=\int_{k_{1}}^{k_{2}} \int_{-\pi}^{\pi} F(k, \phi) e^{j k[c t+x \cos (\theta-\phi)]} k d \phi d k,
$$

where $k=2 \pi f / c, c$ is the speed of wave propagation, $f$ is frequency, and spatial position $\boldsymbol{x}$ is expressed in polar coordinates, $x=|\boldsymbol{x}|$ and $\theta=\angle \boldsymbol{x}$. Extensions to wavefields in 3D space, and source distributions which depend on both azimuth and elevation angle are considered in section 6

In traditional array signal processing, this wavefield is observed using an array of sensors in space, each sampled uniformly and synchronously throughout time. This sampling scheme (shown by sensors $S_{0}$ and $S_{1}$ in Figure 2) can be completely specified by a uniform grid - the Cartesian product of the time and space sampling.

Unfortunately, this simple grid framework is inadequate to deal with more complex space-time sampling schemes (some examples of which are shown by sensors $S_{2}$ and $S_{3}$ in Figure 2). Consider asynchronous sampling where different sensors are sampled at different times, or dynamic arrays where the relative spatial position of sensors changes throughout time. To deal with these complexities, a more general model for space-time sampling is needed which subsumes the traditional Cartesian product framework. An obvious choice is to treat each space-time sample independently. 


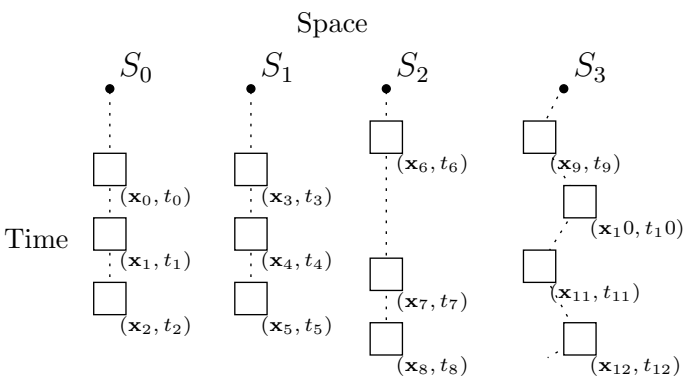

Fig. 1. A space-time sampler with $M=13$ space-time samples which contains uniform synchronous sampling ( $S_{0}$ and $S_{1}$ ), non-uniform asynchronous sampling $\left(S_{2}\right)$, and moving sensors $\left(S_{3}\right)$.

\section{Definition 1. Space-time Sampler}

An $M$ dimensional space-time sampler is defined by a set of unique positions in space and relative time, $\left(\boldsymbol{x}_{m}, t_{m}\right)$ for $m=0, \ldots, M-$ 1. Thus, at some time $t$, the sampler will take $M$ wavefield samples, $f\left(\boldsymbol{x}_{m}, t+t_{m}\right)$.

The traditional model of a broadband beamformer is based on an FIR filter attached to each sensor in space. We generalize this notation to match Definition 1. Thus, any possible linear beamformer can be represented as a linear combination of $M$ independent field observations.

\section{Definition 2. Space-Time Beamformer}

A beamformer for an $M$ element space-time sampler is uniquely specified by an $M$ length vector of complex finite weights $\boldsymbol{w}=$ $\left[w_{0}, \ldots, w_{M-1}\right]^{T}$. The output of this beamformer at some time $t_{s}$ will be

$$
z\left(t_{s}\right)=\sum_{m=0}^{M-1} w_{m} f\left(\boldsymbol{x}_{m}, t_{m}+t_{s}\right) .
$$

Note that this more general model subsumes the traditional FIR filter model. That is, every traditional broadband beamformer can be equivalently expressed in the form of Definition 2 by simply treating each filter coefficient as an independent weighting coefficient.

Combining (1) and (2), the beamformer output can be expressed

$$
z\left(t_{s}\right)=\int_{k_{1}}^{k_{2}} \int_{-\pi}^{\pi} F(k, \phi) W_{\mathrm{ach}}(k, \phi) e^{j k c t_{s}} k d \phi d k
$$

where we have defined an achievable beampattern,

$$
W_{\text {ach }}(k, \phi) \triangleq \sum_{m=0}^{M-1} w_{m} e^{j k\left[c t_{m}+x_{m} \cos \left(\theta_{m}-\phi\right)\right]} .
$$

As long as every space-time sample is unique (that is, no point in space-time is sampled more than once), every achievable beampattern $W_{\text {ach }}(k, \phi)$ will correspond uniquely with a particular vector of weighting coefficients $\boldsymbol{w}$.

Consider some desirable, but perhaps unachievable, beampattern denoted $W_{\text {des }}(k, \phi)$. For a particular space-time sampler, the beamforming problem is to find a set of weightings, $\boldsymbol{w}$, which produce a beampattern, $W_{\text {ach }}(k, \phi)$, as close as possible to the desired pattern. One measure of closeness is the mean-squared error (MSE) between the desired and achievable patterns,

$$
\mathrm{MSE}=\int_{k_{1}}^{k_{2}} \int_{-\pi}^{\pi}\left|W_{\mathrm{des}}(k, \phi)-W_{\mathrm{ach}}(k, \phi)\right|^{2} k d \phi d k .
$$

\section{MODAL SUBSPACE DECOMPOSITION}

\subsection{Operators and Spaces}

In this section, we derive a modal basis for the subspace of achievable beampatterns. The first step is to formally define some vector and function spaces.

Define $\mathcal{S}$, the space of all finite energy weight vectors, and therefore the space of all attainable beamformers,

$$
\mathcal{S} \triangleq\{\boldsymbol{w}:\|\boldsymbol{w}\|<\infty\}
$$

based on the inner product

$$
\langle\boldsymbol{w}, \boldsymbol{y}\rangle_{\mathcal{S}}=\sum_{m=0}^{M-1} w_{m} y_{m}^{*}
$$

and associated norm $\|\boldsymbol{w}\|_{\mathcal{S}}=\sqrt{\langle\boldsymbol{w}, \boldsymbol{w}\rangle_{\mathcal{S}}}$, where ${ }^{*}$ denotes the complex conjugate. $\mathcal{S}$ is an $M$ dimensional complex vector space.

As mentioned in the previous section, the beampattern design process is often based on some desirable beampattern denoted $W_{\text {des }}(k, \phi)$. To formalize this concept, we define $\mathcal{F}$, the space of desired patterns as those patterns with finite energy over the design ranges of $k$ and $\phi$,

$$
\mathcal{F} \triangleq\left\{W_{\text {des }}(k, \phi):\left\|W_{\text {des }}\right\|<\infty\right\},
$$

based on the inner product

$$
\langle W, Y\rangle_{\mathcal{F}}=\int_{k_{1}}^{k_{2}} \int_{-\pi}^{\pi} W(k, \phi) Y^{*}(k, \phi) k d \phi d k,
$$

and associated norm $\|W\|_{\mathcal{F}}=\sqrt{\langle W, W\rangle_{\mathcal{F}}} \cdot \mathcal{F}$ is an infinite dimensional, separable, Hilbert space ${ }^{1}$.

Given a finite dimensional space-time sampler, not all desired beampatterns will be achievable. With this in mind, we can partition $\mathcal{F}$ into two orthogonal subspaces $-\mathcal{W}$ is the space of achievable beampatterns, and $\perp \mathcal{W}$ is the space of unachievable patterns. Now, any desired patterm $W_{\text {des }}(k, \phi) \in \mathcal{F}$ can be expressed,

$$
W_{\mathrm{des}}(k, \phi)=W_{\mathrm{ach}}(k, \phi)+W_{\mathrm{unach}}(k, \phi),
$$

where $W_{\text {ach }}(k, \phi) \in \mathcal{W}$ and $W_{\text {unach }}(k, \phi) \in \perp \mathcal{W}$.

As mentioned earlier, each achievable pattern $W_{\text {ach }}(k, \phi) \in \mathcal{W}$ has a unique mapping with a weighting vector $\boldsymbol{w} \in \mathcal{S}$. Thus, since $\mathcal{S}$ is an $M$ dimensional space, $\mathcal{W}$ must be an $M$ dimensional proper subspace of $\mathcal{F}$. The mapping between the two is defined by an invertible linear operator $A: \mathcal{S} \rightarrow \mathcal{W}$, that projects a set of weighting coefficients to its corresponding achievable beampattern. From (4), the operator is defined by

$$
W_{\mathrm{ach}}(k, \phi)=A \boldsymbol{w}=\sum_{m=0}^{M-1} w_{m} e^{j k\left[c t_{m}+x_{m} \cos \left(\theta_{m}-\phi\right)\right]} .
$$

As $A$ is a bounded, $M$ dimensional operator, there exists an adjoint operator $A^{*}: \mathcal{W} \rightarrow \mathcal{S}$ such that [6],

$$
\left\langle W_{\mathrm{ach}}, A \boldsymbol{y}\right\rangle_{\mathcal{F}}=\left\langle A^{*} W_{\mathrm{ach}}, \boldsymbol{y}\right\rangle_{\mathcal{S}} .
$$

Combining (11) and (12), the adjoint operator is defined as

$$
\boldsymbol{y}=A^{*} W_{\text {ach }}(k, \phi),
$$

${ }^{1} \mathrm{~A}$ Hilbert space is a complete inner product space. Many of the results of linear algebra generalize to linear operations on Hilbert spaces [6]. 
where the elements of $\boldsymbol{y}$ are given by,

$$
\boldsymbol{y}_{m}=\int_{k_{1}}^{k_{2}} \int_{-\pi}^{\pi} W_{\mathrm{ach}}(k, \phi) e^{-j k\left[c t_{m}+x_{m} \cos \left(\theta_{m}-\phi\right)\right]} k d \phi d k \text {. }
$$

\subsection{Modal Bases}

The operators $A^{*} A$ and $A A^{*}$ are known to have some particularly useful properties. Specifically, the $M$ eigenvectors of $A^{*} A$, denoted $\boldsymbol{u}_{n}$, form a complete orthonormal basis for $\mathcal{S}$ (known as vector modes), and the $M$ eigenfunctions of $A A^{*}$, denoted $U_{n}(k, \phi)$, form a complete orthonormal basis for $\mathcal{W}$ (known as continuous modes) [6]. These modes are independent of the wavefield and desired beampattern, and depend only on the geometry of the spacetime sampling. The complete derivation of these modes is presented in [7], and is summarized here.

Expanding the operator $A^{*} A$ using (11) and (14), the vector modes, $\boldsymbol{u}_{n}$, are the solutions to a matrix eigenvector equation of the form

$$
\boldsymbol{Z} \boldsymbol{u}_{n}=\lambda_{n} \boldsymbol{u}_{n} \text { for } n=0, \ldots, M-1,
$$

where the elements of the $M \times M$ matrix $Z$ are given by

$$
\boldsymbol{Z}_{m, m^{\prime}}=2 \pi \int_{k_{1}}^{k_{2}} e^{j k c\left(t_{m^{\prime}}-t_{m}\right)} J_{0}\left(k\left\|\boldsymbol{x}_{m}-\boldsymbol{x}_{m^{\prime}}\right\|\right) k d k .
$$

The real, non-negative eigenvalues are ordered to form a monotonically decreasing series $\lambda_{0} \geq \lambda_{1} \geq \ldots \geq \lambda_{M-1}$. In general, this integral has no closed form solution, but can be calculated numerically to any required degree of accuracy.

Any weighting vector can be expressed as a linear combination of these vector modes,

$$
\boldsymbol{w}=\sum_{n=0}^{M-1}\left\langle\boldsymbol{w}, \boldsymbol{u}_{n}\right\rangle_{\mathcal{S}} \boldsymbol{u}_{n}
$$

From [6], the continuous modes can be derived from their relationship to the vector modes,

$$
\begin{aligned}
U_{n}(k, \phi) & =\frac{1}{\sqrt{\lambda_{n}}} A \boldsymbol{u}_{n} \\
& =\frac{1}{\sqrt{\lambda_{n}}} \sum_{m=0}^{M-1} \boldsymbol{u}_{n, m} e^{j k\left[c t_{m}+x_{m} \cos \left(\theta_{m}-\phi\right)\right]},
\end{aligned}
$$

where $\boldsymbol{u}_{n, m}$ denotes the $m^{\text {th }}$ element of the $n^{\text {th }}$ vector mode. Any achievable beampattern can be expressed as a linear combination of these continuous modes,

$$
W_{\text {ach }}(k, \phi)=\sum_{n=0}^{M-1}\left\langle W_{\text {ach }}, U_{n}\right\rangle_{\mathcal{F}} U_{n}(k, \phi) .
$$

\section{MODAL SUBSPACE DECOMPOSITION (MSD) BEAMFORMING}

Given some desired pattern $W_{\text {des }}(k, \phi) \in \mathcal{F}$, we need to find the achievable pattern $W_{\text {ach }}(k, \phi) \in \mathcal{W}$ which minimizes the MSE as defined in (5). From functional analysis, this optimal pattern will be the orthogonal projection of $W_{\mathrm{des}}(k, \phi)$ onto the subspace $\mathcal{W}[6]$ denoted $B: \mathcal{F} \rightarrow \mathcal{W}$. This projection can be performed by projecting onto the continuous modes,

$$
W_{\text {ach }}(k, \phi)=\sum_{n=0}^{M-1}\left\langle W_{\text {des }}, U_{n}\right\rangle_{\mathcal{F}} U_{n}(k, \phi),
$$

For all but the simplest patterns, this inner product will need to be calculated numerically. Quadrature techniques can be used to calculate the $2 \mathrm{D}$ integral to any desired degree of accuracy with low computational complexity [8].

The modal coefficients $\left\langle W_{\text {des }}, U_{n}\right\rangle_{\mathcal{F}}$ can now be used to find the beamformer weighting coefficients. Combining (11), (18) and (21),

$$
\boldsymbol{w}=\sum_{n=0}^{M-1} \frac{\left\langle W_{\mathrm{des}}, U_{n}\right\rangle_{\mathcal{F}}}{\sqrt{\lambda_{n}}} \boldsymbol{u}_{n} .
$$

\subsection{The MSD Beamforming Algorithm}

In summary, the steps of the algorithm are:

1. Given a set of $M$ space-time sampling positions $\left(\boldsymbol{x}_{m}, t_{m}\right)$, build the matrix $Z$ using (16).

2. Find the eigenvectors, $\boldsymbol{u}_{n}$, and eigenvalues, $\lambda_{n}$, of $\boldsymbol{Z}$, and use (19) to build the continuous modes $U_{n}(k, \phi)$.

3. Given some desired beampattern $W_{\mathrm{des}}(k, \phi)$, the optimal beamformer weighting coefficients can be calculated using (22).

Note that for adaptive beamforming applications, only the third step must be recalculated when the desired pattern changes. The first two steps depend only on the space-time sampling positions, and need only be recalculated if the sampling geometry is changed.

\section{DESIGN EXAMPLES}

Consider an acoustic beamforming environment ( $c=350 \mathrm{~m} / \mathrm{s}$ ) with a design frequency range from $f_{1}=400 \mathrm{~Hz}$ to $f_{2}=4 \mathrm{kHz}$.

\subsection{Frequency-invariant beamforming}

One of the more common problems in broadband beamforming is frequency-invariant beamforming, where the aim is to design a beam with identical angular response across all design frequencies [3,4]. A frequency-invariant 'desired pattern' is created with a beam-centre at $\phi=\pi / 2$, and a beamwidth of $\Delta \phi=\pi / 5$.

Consider a 5 element uniform circular array (UCA) designed for frequency $f_{2}$, with half-wavelength inter-element spacing, and 32 time samples taken at the Nyquist rate. The desired pattern is projected into the modal basis, and the resulting optimal achievable beampattern is shown in Figure 2(a).

One major strength of the MSD technique is that it is equally applicable to sparse arrays. To demonstrate this, a second array with the same spatial radius, but only 3 elements is used. Also, the sampling rate is halved so that only 16 samples are taken over the same time period. Figure 2(b) shows the resulting optimal achievable pattern. Whilst the pattern is clearly inferior to that for the denser array, it is still reasonably directional and frequency invariant.

A comparison with traditional frequency-invariant design techniques is difficult, as these techniques tend to be based on long time sequences, and perform poorly on the short sparse sequences used in this example. Note, though, that since the MSD algorithm optimizes over all possible beamformers, it is impossible for any other design technique to produce an better pattern (in terms of the MSE).

\subsection{Spot beamforming}

Whereas most of the work in broadband beamforming has focussed on frequency-invariant design, the MSD design technique is far more 


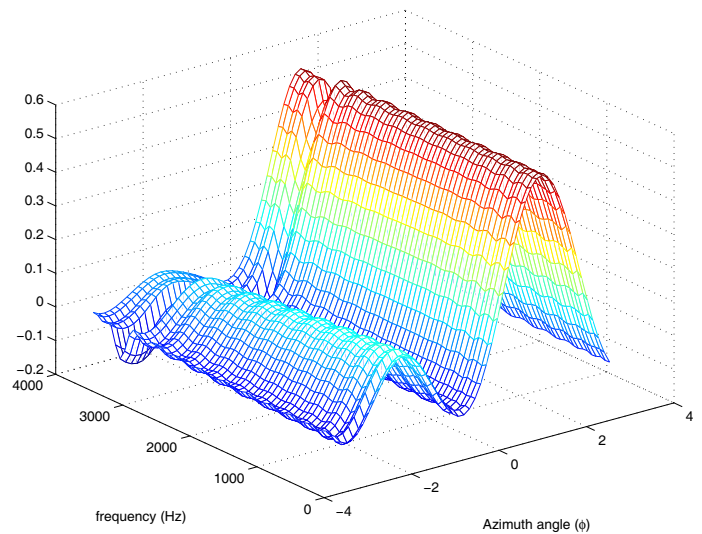

(a) Pattern for 5 element UCA with half-wavelength spacing, and Nyquist sampling (32 time samples).

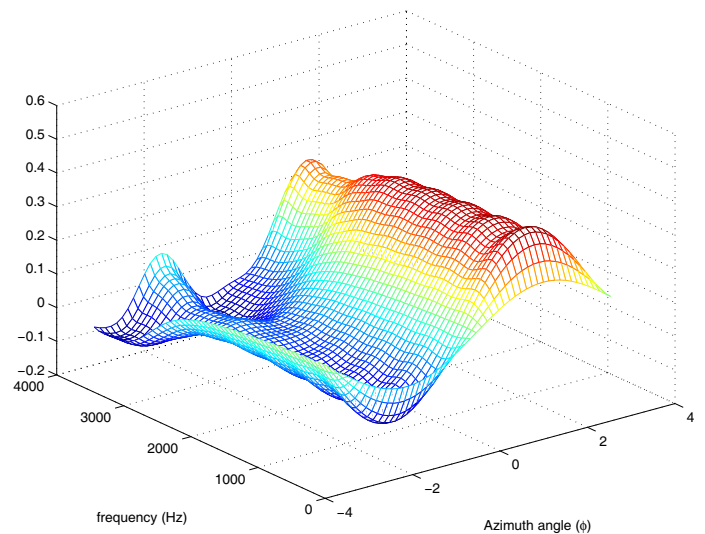

(b) Pattern for UCA with same radius as above, but only 3 sensor elements, and half the sampling rate (16 samples).

Fig. 2. Frequency Invariant Beamforming

versatile. Consider a desired pattern which filters sources based on both frequency and direction - effectively applying different bandpass filters to signals depending on angle of arrival. A desired pattern is developed which focusses at two points: $\phi_{a}=-\pi / 2, f_{a}=1 \mathrm{kHz}$ and $\phi_{b}=\pi / 2, f_{b}=3 \mathrm{kHz}$ (with a spot-width of $\Delta \phi=\pi / 5$ and $\Delta f=400 \mathrm{~Hz}$ ). Projecting this pattern into the modal basis for the 5 element, 32 sample array used in the previous example results in the achievable pattern shown in Figure 3. The ability to design complex patterns is an advantage over many previous, more specialized, techniques.

\section{EXTENSIONS}

Although the MSD technique has been presented in the context of the broadband farfield beamforming problem, the same basic concept can easily be applied to more complex problems. Equation (1), which maps a farfield source distribution to a 2D wavefield, can be altered to model more complex mappings such as nearfield sources, sources distributed in both azimuth and elevation angle, or wavefields in $3 \mathrm{D}$ space. Although this altered mapping will change the form of the modal basis, the same basic methods and derivations can be applied. These extensions will be explored in a future paper.

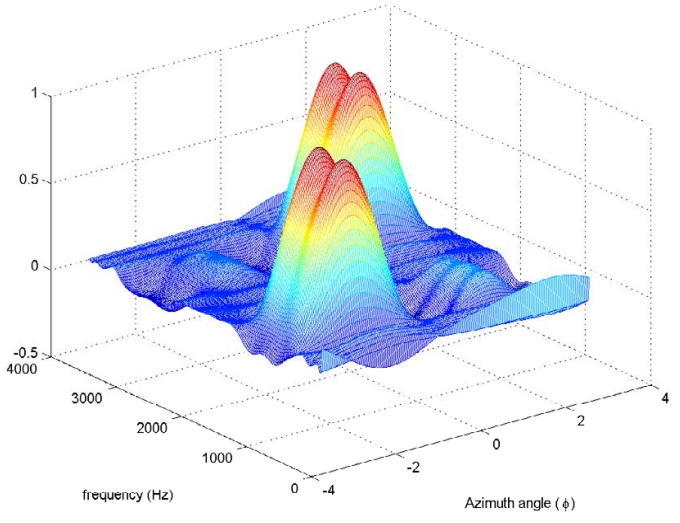

Fig. 3. Spot beamforming - Optimal Achieved Pattern

\section{CONCLUSION}

A new technique for broadband farfield beamforming has been proposed which produces an optimal beamformer for any set of spacetime sampling positions. The main advantage of the technique is versatility as it can produce optimal beamformers for both sparse and dense arrays, non-uniform and asynchronous time sampling, and dynamic arrays where sensors can move throughout space. Design examples were presented to show that the technique is applicable to both frequency-invariant and spot beamforming.

\section{REFERENCES}

[1] B. D. Van-Veen and K. M. Buckley, "Beamforming: a versatile approach to spatial filtering," IEEE ASSP Magazine, vol. 5, no. 2, pp. 4-24, 1988.

[2] L. C. Godara, "Application of the fast fourier transform to broadband beamforming," J. Acoust. Soc. Amer, vol. 98, no. 1, pp. 230-240, 1995.

[3] D. B. Ward, R. A. Kennedy, and R. C. Williamson, "Theory and design of broadband sensor arrays with frequency invariant far-field beampatterns," J. Acoust. Soc. Amer, vol. 97, pp. 10231034, 1995.

[4] Wei Liu and S. Weiss, "New class of broadband arrays with frequency invariant beam patterns," in Proc. ICASSP., 2004, vol. 2, pp. ii $-185-8$ vol.2.

[5] M. A. Poletti, "A unified theory of horizontal holographic sound systems," Journal of the Audio Engineering Society, vol. 48, no. 12, pp. 1155-1182, 2000.

[6] L. Debnath and P. Mikusinski, Introduction to Hilbert Spaces with Applications, Academic Press, San Diego, CA, 1990.

[7] M. I. Y. Williams, T. D. Abhayapala, and R. A. Kennedy, "Generalized broadband beamforming using a modal subspace decomposition," submitted to EURASIP Journal on Applied Signal Processing, preprint available at http://users.rsise. anu. edu.au/ mickw/bf.pdf.

[8] A. H. Stroud, Approximate calculation of multiple integrals, Prentice-Hall, New Jersey, 1971. 